PROCEEDINGS OF THE

AMERICAN MATHEMATICAL SOCIETY

Volume 135, Number 10, October 2007, Pages 3383-3391

S 0002-9939(07)08845-4

Article electronically published on May 14, 2007

\title{
TURING DEGREES OF NONABELIAN GROUPS
}

\author{
M. A. DABKOWSKA, M. K. DABKOWSKI, V. S. HARIZANOV, AND A. S. SIKORA
}

(Communicated by Julia Knight)

\begin{abstract}
For a countable structure $\mathcal{A}$, the (Turing) degree spectrum of $\mathcal{A}$ is the set of all Turing degrees of its isomorphic copies. If the degree spectrum of $\mathcal{A}$ has the least degree $\mathbf{d}$, then we say that $\mathbf{d}$ is the (Turing) degree of the isomorphism type of $\mathcal{A}$. So far, degrees of the isomorphism types have been studied for abelian and metabelian groups. Here, we focus on highly nonabelian groups. We show that there are various centerless groups whose isomorphism types have arbitrary Turing degrees. We also show that there are various centerless groups whose isomorphism types do not have Turing degrees.
\end{abstract}

\section{Introduction, PRELIMINARIES, AND NOtation}

One of the goals of computable algebra is to use the tools and methods of computability theory to measure the complexity of algebraic notions and constructions. While in algebra we identify two isomorphic structures, from the point of view of computable algebra, they can have very different computability-theoretic properties. In particular, two countable isomorphic structures can have distinct Turing degrees. Since the Turing degree of a structure is not invariant under isomorphism, Jockusch and Richter (see [27]) introduced the following complexity measure of the isomorphism type of a structure. To a countable structure $\mathcal{A}$, they assigned the least Turing degree among all Turing degrees of the isomorphic copies of $\mathcal{A}$ and called it the degree of the isomorphism type of $\mathcal{A}$.

We will now give exact definitions and specify notation. We consider only countable structures for computable (mainly finite) languages. The universe $A$ of an infinite countable structure $\mathcal{A}$ can be identified with the set of natural numbers $\omega$. If $L$ is the language of $\mathcal{A}$, then $L_{A}$ is the language $L$ expanded by adding a constant symbol a for every $a \in A$, and $\mathcal{A}_{A}=(\mathcal{A}, a)_{a \in A}$ is the corresponding expansion of $\mathcal{A}$ to $L_{A}$. The atomic diagram, or the open diagram, of a structure $\mathcal{A}, D^{a}(\mathcal{A})$, is the set of all quantifier-free sentences of $L_{A}$ true in $\mathcal{A}_{A}$. The elementary diagram of $\mathcal{A}, D^{e}(\mathcal{A})$, is the set of all sentences of $L_{A}$, which are true in $\mathcal{A}_{A}$. A structure $\mathcal{A}$ is computable if $D^{a}(\mathcal{A})$ is computable. The Turing degree of $\mathcal{A}, \operatorname{deg}(\mathcal{A})$, is the Turing degree of $D^{a}(\mathcal{A})$. Hence $\mathcal{A}$ is computable iff $\operatorname{deg}(\mathcal{A})=\mathbf{0}$. Clearly, every finite structure is computable. A structure for a finite language is computable if its

Received by the editors March 21, 2006 and, in revised form, July 1, 2006.

2000 Mathematics Subject Classification. Primary 03C57, 03D45.

Key words and phrases. Computable structure, Turing degree, degree spectrum, isomorphism, group. 
domain is a computable set and its operations and relations are computable. (For more on computable structures, see [3] and [10.)

Obviously, if there is a computable structure in the isomorphism class of a structure, then the Turing degree of the isomorphism type of the structure is $\mathbf{0}$. However, the degree of the isomorphism type of a structure does not always exist (see Richter [27]). Indeed, Slaman [30] and Wehner [32] independently showed that there is a structure with isomorphic copies in every nonzero Turing degree, but without a computable isomorphic copy. While Wehner's structure is elementarily equivalent to a computable structure, Slaman's structure is not. Recently, Hirschfeldt [14] showed that such a structure can be a prime model of a complete decidable theory.

Here, we focus on the isomorphism types of countable groups. Khisamiev [17] showed that every abelian $p$-group ( $p$ is a prime number) without a computable isomorphic copy does not have a degree of its isomorphism type. On the other hand, Richter [27] showed that for every Turing degree $\mathbf{d}$, there is a torsion (that is, with all elements of finite order) abelian group whose isomorphism type has degree $\mathbf{d}$. However, she also established that there is a torsion abelian group whose isomorphism type does not have a degree.

Downey and Knight (see 7) showed that for any Turing degree $\mathbf{d}$, there is a torsion-free abelian group of rank 1 whose isomorphism type has degree $\mathbf{d}$. Also, Downey and Jockusch (see [7) showed that some abelian torsion-free rank 1 groups do not have a degree of the isomorphism type. Calvert, Harizanov and Shlapentokh [5] recently proved that there are torsion-free abelian groups of any finite rank whose isomorphism types have arbitrary Turing degrees, as well as torsion-free abelian groups of any finite rank whose isomorphism types fail to have a Turing degree. Calvert, Harizanov and Shlapentokh [5] also obtained similar results for (countable) fields and rings of algebraic numbers and functions. Hirschfeldt, Khoussainov, Shore and Slinko [15] established a general result from which it follows that there is a 2step nilpotent group, also called metabelian (see [28]), with an arbitrary Turing degree of its isomorphism type, as well as a metabelian group without a degree of its isomorphism type.

For those countable structures that fail to have a degree of the isomorphism type, Jockusch introduced Turing jump degrees. For a (countable) structure $\mathcal{A}$ and a computable ordinal $\alpha$, the $\alpha$ th jump degree of $\mathcal{A}$, if it exists, is the least Turing degree in the set of the Turing degrees of the $\alpha$ th Turing jumps of the atomic diagrams of all isomorphic copies of $\mathcal{A}$. Thus, the 0th jump degree of $\mathcal{A}$ is the degree of the isomorphism type of $\mathcal{A}$. By considering jump degrees, we can assign Turing degrees to some isomorphism types that do not have degrees. For example, Richter [27] showed that a linear ordering, which is not isomorphic to a computable one, does not have a degree of its isomorphism type. Downey and Knight 8, building on the previous work of Knight [18 and Ash, Jockusch and Knight [2, established that for a Turing degree $\mathbf{d}$ with $\mathbf{d} \geq \mathbf{0}^{(\alpha)}$, where $\alpha \geq 1$ is a computable ordinal, there is a linear ordering $\mathcal{A}$ whose $\alpha$ th jump degree is $\mathbf{d}$, and such that $\mathcal{A}$ does not have $\beta$ th jump degree for any $\beta<\alpha$. The jump degrees have also been studied for torsion abelian groups by Oates [24, and for rank 1 torsion-free abelian groups by Coles, Downey and Slaman 6. In addition, Turing degrees and jump degrees of the isomorphism types have been studied for partial orderings, trees, Boolean algebras, models of Peano Arithmetic, and prime models (for a survey of these results, see [11]). 
So far, all groups whose isomorphism type degrees have been studied are abelian or metabelian. Here, we investigate Turing degrees of the isomorphism types for various "highly nonabelian" groups. In particular, since our groups are obtained using nontrivial free products, they are centerless, hence nonnilpotent.

For a group $\mathcal{G}$, we call a pair $\langle U \mid R\rangle$ a presentation of $\mathcal{G}$ and write $\mathcal{G}=\langle U \mid R\rangle$ if $\mathcal{G}$ is the quotient group of a free group $\mathcal{F}$ with the basis $U$ by the normal closure in $\mathcal{F}$ of the set $R$. The elements of $U$ are called the generators of $\mathcal{G}$, and the elements of $R$ the relators of $\mathcal{G}$. The equations $r=e$ for $r \in R$, where $e$ is the identity element, are the relations of $\mathcal{G}$. We say that $\mathcal{G}$ is finitely generated if $U$ is finite, and that $\mathcal{G}$ is finitely presented if both $U$ and $R$ are finite. A finitely generated group $\mathcal{G}$ has a decidable word problem with respect to its generators $g_{1}, \ldots, g_{n}$ if the set of words $w\left(x_{1}, \ldots, x_{n}\right)$ on the symbols $x_{1}, \ldots, x_{n}$ for which the equation

$$
w\left(x_{1}, \ldots, x_{n}\right)=e
$$

is satisfied in $\mathcal{G}$ upon substituting $g_{i}$ for $x_{i}, i \in\{1, \ldots, n\}$, is a computable subset of all words on $x_{1}, \ldots, x_{n}$. Rabin [26] showed that the decidability of the word problem of $\mathcal{G}$ does not depend on the system of generators. Rabin also showed that a finitely generated group $\mathcal{G}$ has a decidable word problem if and only if $\mathcal{G}$ has a computable isomorphic copy.

Let $\left\{\mathcal{G}_{i}: i \in I\right\}$ be a family of groups. A free product of these groups is a group $\mathcal{G}$, in symbols $\mathcal{G}=\star_{i \in I} \mathcal{G}_{i}$, which has the following properties:

(i) $\mathcal{G}$ contains an isomorphic copy of each $\mathcal{G}_{i}$,

(ii) for every group $\mathcal{B}$ and every family of homomorphisms $f_{i}: G_{i} \rightarrow B, i \in I$, there is a unique homomorphism $h: G \rightarrow B$ extending each $f_{i}$.

The next fundamental result about free products is known as the Kurosh Subgroup Theorem (see [20]).

Theorem 1.1. Let $\mathcal{G}=\star_{i \in I} \mathcal{G}_{i}$. Let $\mathcal{H}$ be a subgroup of $\mathcal{G}$. Then $\mathcal{H}$ is the free product of a free group together with groups that are conjugates of subgroups of the free factors $\mathcal{G}_{i}$ of $\mathcal{G}$.

It follows that $\mathcal{H}$ is isomorphic to the free product of a free group together with groups isomorphic to subgroups of $\mathcal{G}_{i}$ 's.

For a function $f$, by $\operatorname{ran}(f)$ we denote its range. We use $\leq_{T}$ for Turing reducibility and $\equiv_{T}$ for Turing equivalence of sets. By $\operatorname{deg}(X)$ we denote the Turing degree of $X$. For a structure $\mathcal{A}$, we often abbreviate $\operatorname{deg}(\mathcal{A}) \leq \operatorname{deg}(Y)$ by $\mathcal{A} \leq_{T} Y$. For $X, Y \subseteq \omega$, the join of $X$ and $Y$ is

$$
X \oplus Y=\{2 m: m \in X\} \cup\{2 m+1: m \in Y\} .
$$

We denote the set of all Turing degrees by $\mathcal{D}$. It has cardinality $2^{\aleph_{0}}$. The partially ordered set $(\mathcal{D}, \leq)$ forms an upper semilattice in which the supremum of $\operatorname{deg}(X)$ and $\operatorname{deg}(Y)$ is $\operatorname{deg}(X \oplus Y)$. This semilattice is not a lattice.

For $X, Y \subseteq \omega$, we say that $X$ is enumeration reducible to $Y$, in symbols $X \leq_{e} Y$, if there is a computably enumerable (abbreviated by c.e.) binary relation $E$ such that

$$
x \in X \Leftrightarrow \exists u\left[D_{u} \subseteq Y \wedge E(x, u)\right],
$$

where $D_{u}$ is the finite set with canonical index $u$. Intuitively, $X \leq_{e} Y$ iff for every set $S$, if $Y$ is c.e. relative to $S$, then $X$ is c.e. relative to $S$. For additional information on Turing and enumeration degrees, see Soare [31] and Odifreddi [25]. 


\section{TURING DEGREES OF ISOMORPHISM TYPES OF STRUCTURES}

The Turing degree spectrum of a (countable) structure $\mathcal{A}$ is

$$
\operatorname{DgSp}(\mathcal{A})=\{\operatorname{deg}(\mathcal{B}): \mathcal{B} \cong \mathcal{A}\} .
$$

A structure $\mathcal{M}$ is automorphically trivial if there is a finite subset $P$ of the domain $M$ such that every permutation of $M$, whose restriction to $P$ is the identity, is an automorphism of $\mathcal{M}$. Knight [18] proved that for an automorphically nontrivial structure $\mathcal{A}$, and a Turing degree $\mathbf{x}$ with $\mathbf{x} \geq \operatorname{deg}(\mathcal{A})$, there is a structure $\mathcal{B} \cong \mathcal{A}$ such that $\operatorname{deg}(\mathcal{B})=\mathbf{x}$. That is, $\operatorname{DgSp}(\mathcal{A})$ is closed upwards. On the other hand, for an automorphically trivial structure, all isomorphic copies have the same Turing degree, and in the case of a finite language that degree must be $\mathbf{0}$ (see [13]). Harizanov, Knight and Morozov 12 showed that, while for every automorphically trivial structure $\mathcal{M}$ we have $D^{e}(\mathcal{M}) \equiv_{T} D^{a}(\mathcal{M})$, for every automorphically nontrivial structure $\mathcal{A}$ and every set $X \geq_{T} \quad D^{e}(\mathcal{A})$, there exists $\mathcal{B} \cong \mathcal{A}$ such that $D^{e}(\mathcal{B}) \equiv_{T} D^{a}(\mathcal{B}) \equiv_{T} X$.

Now, the Jockusch-Richter definition can be stated as follows.

Definition 2.1. The Turing degree of the isomorphism type of $\mathcal{A}$, if it exists, is the least Turing degree in $\operatorname{Dg} S p(\mathcal{A})$.

Richter [27] established the following general criterion for the existence of a structure whose isomorphism type has an arbitrary Turing degree. We will write $\mathcal{A} \hookrightarrow \mathcal{B}$ if $\mathcal{A}$ is embeddable in $\mathcal{B}$.

Theorem 2.2. Let $T$ be a theory in a finite language $L$ such that there is a computable sequence

$$
\mathcal{A}_{0}, \mathcal{A}_{1}, \mathcal{A}_{2}, \ldots
$$

of finite structures for $L$ that are pairwise nonembeddable. Assume that for every set $X \subseteq \omega$, there is a (countable) model $\mathcal{A}_{X}$ of $T$ such that

$$
\mathcal{A}_{X} \leq_{T} X,
$$

and for every $i \in \omega$,

$$
\mathcal{A}_{i} \hookrightarrow \mathcal{A}_{X} \Leftrightarrow i \in X
$$

Then for every Turing degree $\mathbf{d}$, there is a model of $T$ whose isomorphism type has degree $\mathbf{d}$.

For example, Richter used Theorem 2.2 to prove that for every Turing degree $\mathbf{d}$, there is a torsion abelian group whose isomorphism type has degree $\mathbf{d}$.

On the other hand, Richter 27] showed that a modification of Theorem [2.2, obtained by replacing Turing reducibility in $\mathcal{A}_{X} \leq_{T} X$ by the enumeration reducibility $\mathcal{A}_{X} \leq_{e} X$, yields a very different conclusion - that there is a set $Y$ such that the isomorphism type of $\mathcal{A}_{Y}$ does not have a degree. As a corollary, Richter proved that there is a (countable) torsion abelian group whose isomorphism type does not have a degree.

\section{TURING DEGREES OF ISOMORPHISM TYPES OF CENTERLESS GROUPS}

We will now apply Richter's theorems to study the Turing degrees of the isomorphism types of various nonabelian groups.

For a group $\mathcal{G}$, its center $Z(\mathcal{G})$ is defined by:

$$
Z(\mathcal{G})=\{z \in G:(\forall g \in G)[z g=g z]\} .
$$


A group $\mathcal{G}$ is centerless if $Z(\mathcal{G})=\{e\}$. Clearly, nontrivial centerless groups are nonnilpotent. A free product of nontrivial groups produces infinite centerless groups. We will now state the following well-known group-theoretic lemma. For completeness we will also sketch its proof.

Lemma 3.1. Let $p$ and $q$ be primes such that $q \mid(p-1)$. Then there is a nonabelian group of order $p q$.

Proof. Let $\left(\mathbb{Z}_{q},+\right)$ be the additive cyclic group with $q$ elements, where, as usual, $\mathbb{Z}_{q}=\{0,1, \ldots, q-1\}$. Let $\left(\mathbb{Z}_{p}^{*}, \cdot\right)$ be the multiplicative group of the field $\left(\mathbb{Z}_{p},+, \cdot\right)$. Consider an embedding

$$
\tau:\left(\mathbb{Z}_{q},+\right) \hookrightarrow\left(\mathbb{Z}_{p}^{*}, \cdot\right) .
$$

Such an embedding $\tau$ exists since $\left(\mathbb{Z}_{p}^{*}, \cdot\right)$ is cyclic. Let $\mathbb{G}(q, p)$ be the semidirect product of $\left(\mathbb{Z}_{q},+\right)$ and $\left(\mathbb{Z}_{p},+\right)$. That is, for $(a, b),(c, d) \in \mathbb{Z}_{q} \times \mathbb{Z}_{p}$, we have in $\mathbb{G}(q, p)$ :

$$
(a, b)(c, d)=(a+c, b+\tau(a) \cdot d) .
$$

The group $\mathbb{G}(q, p)$ is nonabelian and of order $p q$.

Theorem 3.2. For every Turing degree $\mathbf{d}$, there is a centerless group $\mathcal{A}$ whose isomorphism type has degree $\mathbf{d}$ and

$$
\operatorname{Dg} S p(\mathcal{A})=\{\mathbf{x} \in \mathcal{D}: \mathbf{x} \geq \mathbf{d}\} .
$$

Furthermore, $\mathcal{A}$ has infinitely many nonabelian finite subgroups.

Proof. Let

$$
p_{0}=3, p_{1}=5, p_{2}=7, \ldots
$$

be the increasing sequence of all primes $>2$. For every $i \in \omega$, let $q_{i}$ be the greatest prime divisor of $p_{i}-1$, and let $\mathcal{A}_{i}=\mathbb{G}\left(q_{i}, p_{i}\right)$. The sequence $\left(\mathcal{A}_{i}\right)_{i \in \omega}$ consists of pairwise nonembeddable groups, since for primes $p_{i}, q_{i}, p_{j}, q_{j}$ with $q_{i}<p_{i}$ and $q_{j}<p_{j}$, we have

$$
\left(p_{i} q_{i} \mid p_{j} q_{j}\right) \Leftrightarrow\left(p_{i}=p_{j} \wedge q_{i}=q_{j}\right) .
$$

Let $X \subseteq \omega$. Let $\mathcal{A}_{X}$ be the free product of the groups $\mathcal{A}_{i}$ for $i \in X$ :

$$
\mathcal{A}_{X}=\underset{i \in X}{*} \mathcal{A}_{i}
$$

We can arrange that $\mathcal{A}_{X}$ is a group with domain $\omega$ such that $\mathcal{A}_{X} \leq_{T} X$. Clearly, if $i \in X$, then $\mathcal{A}_{i} \hookrightarrow \mathcal{A}_{X}$. Conversely, for some $k \in \omega$, let $f$ be an embedding from $\mathcal{A}_{k}$ into $\mathcal{A}_{X}$. Consider $f\left(\mathcal{A}_{k}\right)$. Since $f\left(\mathcal{A}_{k}\right)$ is a subgroup of $\mathcal{A}_{X}$ consisting of the elements of finite order, by the Kurosh Subgroup Theorem, it is a conjugate of some subgroup of $\mathcal{A}_{i}$ for $i \in X$. Hence $k=i$, so $k \in X$.

Let $\mathcal{A}=\mathcal{A}_{X}$, where $X=D \oplus \bar{D}$ and $D \subseteq \omega$ is an infinite set of Turing degree d. Since $\mathcal{A}$ is automorphically nontrivial, we have that $\operatorname{Dg} \operatorname{Sp}(\mathcal{A})=$ $\{\mathbf{x} \in \mathcal{D}: \mathbf{x} \geq \mathbf{d}\}$.

We can further obtain the following result by using a proof similar to the one of the previous theorem, and by choosing $X \subseteq \omega$ so that the set of functions $\{f: \operatorname{ran}(f)=X\}$ has no Turing least element. (See Theorem 2.3 in Richter [27].)

Theorem 3.3. There is a centerless group with infinitely many finite nonabelian subgroups such that its isomorphism type does not have a Turing degree. 
We can also apply a free product construction, similar to the one in the proof of Theorem 3.2, to the sequence of all finite cyclic groups of prime order

$$
\mathbb{Z}_{2}, \mathbb{Z}_{3}, \mathbb{Z}_{5}, \mathbb{Z}_{7}, \ldots
$$

We then get the following result.

Theorem 3.4. (i) For every Turing degree $\mathbf{d}$, there is a centerless group $\mathcal{A}$ without infinite noncyclic abelian subgroups, all of whose finite subgroups are cyclic, such that the isomorphism type of $\mathcal{A}$ has degree $\mathbf{d}$.

(ii) There is a centerless group $\mathcal{B}$ without infinite noncyclic abelian subgroups, all of whose finite subgroups are cyclic, such that the isomorphism type of $\mathcal{B}$ has no degree.

Next, we will prove a general result, which is a modification of Theorem 2.2, but allows infinite structures in the antichain of structures.

Theorem 3.5. Let $\mathcal{C}$ be a class of countable structures for a finite language $L$, closed under isomorphisms. Assume that there is a computable sequence $\mathcal{A}_{0}, \mathcal{A}_{1}$, $\mathcal{A}_{2}, \ldots$ of (possibly infinite) pairwise nonembeddable structures for $L$ such that for every set $X \subseteq \omega$, there is a structure $\mathcal{A}_{X}$ in $\mathcal{C}$ satisfying the following conditions.

(i) $\mathcal{A}_{X} \leq_{T} X$.

(ii) For every $i \in \omega$,

$$
\mathcal{A}_{i} \hookrightarrow \mathcal{A}_{X} \Leftrightarrow i \in X .
$$

(iii) Suppose that $\mathcal{A}_{X}$ is isomorphic to a structure $\mathcal{B}$. Then there is a uniformly effective procedure with oracle $\mathcal{B}$, which for a pair of structures $\mathcal{A}_{i}, \mathcal{A}_{j}$ such that exactly one of them embeds in $\mathcal{B}$, decides which of the two structures embeds in $\mathcal{B}$.

Then for every Turing degree $\mathbf{d}$, there is a structure in $\mathcal{C}$ whose isomorphism type has degree $\mathbf{d}$.

Proof. Let $D \subseteq \omega$ be such that $\operatorname{deg}(D)=\mathbf{d}$. We will show that $\mathcal{A}_{D \oplus \bar{D}}$ is a structure in $\mathcal{C}$, whose isomorphism type has Turing degree $\mathbf{d}$. Clearly,

$$
\operatorname{deg}\left(\mathcal{A}_{D \oplus \bar{D}}\right) \leq \operatorname{deg}(D \oplus \bar{D})=\mathbf{d} .
$$

Now, let a structure $\mathcal{B}$ be such that $\mathcal{B} \simeq \mathcal{A}_{D \oplus \bar{D}}$. We then have, by the definition of $D \oplus \bar{D}$ and the assumption (ii) of the theorem, that for every $j \in \omega$ :

$$
\begin{gathered}
\left(j \in D \Leftrightarrow \mathcal{A}_{2 j} \hookrightarrow \mathcal{B}\right), \text { and } \\
\left(j \notin D \Leftrightarrow \mathcal{A}_{2 j+1} \hookrightarrow \mathcal{B}\right) .
\end{gathered}
$$

Thus, by the assumption (iii) of the theorem, we conclude that $D \leq_{T} \mathcal{B}$. Hence $\operatorname{deg}\left(\mathcal{A}_{D \oplus \bar{D}}\right)=\mathbf{d}$, and the degree of the isomorphism type of $\mathcal{A}_{D \oplus \bar{D}}$ is $\mathbf{d}$.

We can also establish the following result "dual" to Theorem 3.5, which we state without proof.

Theorem 3.6. Let $\mathcal{C}$ be a class of countable structures for a finite language $L$, closed under isomorphisms. Assume that there is a computable sequence $\mathcal{A}_{0}, \mathcal{A}_{1}$, $\mathcal{A}_{2}, \ldots$ of (possibly infinite) pairwise nonembeddable structures for $L$ such that for every set $X \subseteq \omega$, there is a structure $\mathcal{A}_{X}$ in $\mathcal{C}$ satisfying the following conditions.

(i) $\mathcal{A}_{X} \leq_{e} X$.

(ii) For every $i \in \omega$,

$$
\mathcal{A}_{i} \hookrightarrow \mathcal{A}_{X} \Leftrightarrow i \in X
$$


(iii) Suppose that $\mathcal{A}_{X}$ is isomorphic to a structure $\mathcal{B}$. Then from any enumeration of $\mathcal{B}$, we can effectively enumerate those $i$ for which $\mathcal{A}_{i} \hookrightarrow \mathcal{B}$.

Then there is a set $Y \subseteq \omega$ such that the isomorphism type of $\mathcal{A}_{Y}$ has no Turing degree.

Next, we will apply the previous two theorems to a very interesting class of nonabelian groups. We first introduce the following definition.

Definition 3.7. A group $\mathcal{G}$ is of a finite exponent if there is a finite positive integer $n$ such that $g^{n}=e$ for all $g \in G$. If, in addition, there is no positive integer $m<n$ such that $g^{m}=e$ for all $g \in G$, then we say that $G$ has the exponent $n$.

Burnside 4 was the first to consider groups $\mathcal{G}$ of finite exponents. In particular, he asked whether there exists an infinite and finitely generated group $\mathcal{G}$ of finite exponent. This question became known as the Burnside Problem.

Let $F_{r}=\left\langle x_{1}, x_{2}, \ldots, x_{r} \mid-\right\rangle$ be a free group of rank $r$ and let $B(r, n)=F_{r} / N$, where $N$ is the normal subgroup of $F_{r}$ generated by $\left\{g^{n}: g \in F_{r}\right\}$. We call $B(r, n)$ the rank $r$ Burnside group of exponent $n$. Thus, Burnside's question can be formulated as: For what values of $r$ and $n$ is $B(r, n)$ infinite?

The group $B(r, n)$ is finite when $r=1$, or $r$ is an arbitrary positive integer and $n=2,3,4,6$ (see [4, 19, 29, 9]). It was proved by Novikov and Adjan (21, 22, 23]) that $B(r, n)$ is infinite when $r>1, n$ is odd, and $n \geq 4381$. This result was later improved by Adjan 11, who showed that $B(r, n)$ is infinite if $r>1, n$ is odd, and $n \geq 665$. For these $r$ and $n$, Novikov's and Adjan's proof implies that any finite or abelian subgroup of $B(r, n)$ is cyclic, as well as that the word problem for $B(r, n)$ is decidable (see [16]). Hence every such group has a computable copy. (It is still open, for example, whether $B(2,5)$, or $B(2,7)$, is infinite.)

Let the sequence $\left(\hat{p}_{i}\right)_{i \in \omega}$ be the increasing sequence of all prime numbers $\geq 665$. Let $\mathcal{G}_{i}, i \in \omega$, be a computable group such that

$$
\mathcal{G}_{i} \simeq B\left(r, \hat{p}_{i}\right),
$$

where $r \geq 2$ is fixed, and $i \in \omega$. The following lemma establishes an important property for the sequence $\left(\mathcal{G}_{i}\right)_{i \in \omega}$.

Lemma 3.8. Let $i, j \in \omega$. Then $\mathcal{G}_{i} \hookrightarrow \mathcal{G}_{j}$ iff $i=j$.

Proof. First observe that every element of $\mathcal{G}_{i} \simeq B\left(r, \hat{p}_{i}\right)$ has a fixed prime order. Hence $\mathcal{G}_{i} \hookrightarrow \mathcal{G}_{j}$ if and only if $\mathcal{G}_{j}$ has an element of order $\hat{p}_{i}$. Since $\hat{p}_{i}$ is prime, we have that $\hat{p}_{i}=\hat{p}_{j}$, and thus $i=j$.

It follows from the Torsion Theorem for Free Products (see [20]) that an element of finite order in the free product of groups is a conjugate of an element of finite order in one of the factors.

Corollary 1. Let $X \subseteq \omega$, and let $\mathcal{B} \simeq \underset{j \in X}{*} \mathcal{G}_{j}$. Then $\mathcal{G}_{i} \hookrightarrow \underset{j \in X}{*} \mathcal{G}_{j}$ iff $i \in X$. Moreover, there is a uniformly effective procedure with oracle $\mathcal{B}$, which for a pair of groups $\mathcal{G}_{i}, \mathcal{G}_{j}$ such that exactly one of them embeds in $\mathcal{B}$, decides which of the two groups embeds in $\mathcal{B}$. Also, from any enumeration of $\mathcal{B}$, we can effectively enumerate those $i$ for which $\mathcal{G}_{i} \hookrightarrow \mathcal{B}$.

We are now ready to apply Theorems 3.5 and 3.6 to the sequence $\left(\mathcal{G}_{i}\right)_{i \in \omega}$ to obtain the following result. 
Theorem 3.9. For every Turing degree $\mathbf{d}$, there is a centerless group $\mathcal{A}$ whose isomorphism type has degree $\mathbf{d}$, such that $\mathcal{A}$ has infinitely many infinite nonabelian subgroups, which are generated by a fixed finite number of generators, and are of finite exponents. In addition, every finite or abelian subgroup of $\mathcal{A}$ is cyclic. Furthermore, there is also such a centerless group $\mathcal{C}$ whose isomorphism type has no degree.

\section{ACKNowledgments}

Harizanov was partially supported by the NSF grant DMS-0502499 and by the Columbian Research Fellowship of GWU. She thanks William Frawley for strong research support.

\section{REFERENCES}

[1] S. I. Adjan, The Burnside Problem and Identities in Groups, Nauka, Moscow, 1975 (in Russian). (Translated by J. Lennox and J. Wiegold, Ergebnisse der Mathematik und ihrer Grenzgebiete 95, Springer-Verlag, Berlin, 1979.) MR0537580 (80d:20035)

[2] C. J. Ash, C. G. Jockusch, Jr. and J. F. Knight, Jumps of orderings, Transactions of the American Mathematical Society 319 (1990), pp. 573-599. MR0955487 (90j:03081)

[3] C. J. Ash and J. F. Knight, Computable Structures and the Hyperarithmetical Hierarchy, Elsevier, Amsterdam, 2000. MR.1767842 (2001k:03090)

[4] W. Burnside, On an unsettled question in the theory of discontinuous groups, Quarterly Journal of Pure and Applied Mathematics 33 (1902), pp. 230-238.

[5] W. Calvert, V. Harizanov and A. Shlapentokh, Turing degrees of the isomorphism types of algebraic objects, to appear in the Journal of the London Mathematical Society.

[6] R. J. Coles, R. G. Downey and T. A. Slaman, Every set has a least jump enumeration, Journal of the London Mathematical Society 62 (2000), pp. 641-649. MR.1794274 (2002a:03081)

[7] R. Downey, On presentations of algebraic structures, in: A. Sorbi, editor, Complexity, Logic and Recursion Theory, Lecture Notes in Pure and Applied Mathematics 197 (Marcel Dekker, New York), 1997, pp. 157-205. MR1455136 (98k:03099)

[8] R. Downey and J. F. Knight, Orderings with $\alpha$ th jump degree $\mathbf{0}^{(\alpha)}$, Proceedings of the American Mathematical Society 114 (1992), pp. 545-552. MR1065942 (92e:03063)

[9] M. Hall, Jr., Solution of the Burnside problem for exponent six, Illinois Journal of Mathematics 2 (1958), pp. 764-786. MR0102554(21:1345)

[10] V. S. Harizanov, Pure computable model theory, Chapter 1, in: Yu. L. Ershov, S. S. Goncharov, A. Nerode and J. B. Remmel, editors, Handbook of Recursive Mathematics, vol. 1 (Elsevier, Amsterdam, 1998), pp. 3-114. MR.1673621 (2000f:03108)

[11] V. S. Harizanov, Computability-theoretic complexity of countable structures, Bulletin of Symbolic Logic 8 (2002), pp. 457-477. MR1956865(2004h:03076)

[12] V. S. Harizanov, J. F. Knight and A. S. Morozov, Sequences of $n$-diagrams, Journal of Symbolic Logic 67 (2002), pp. 1227-1247. MR1926609 (2003f:03046)

[13] V. Harizanov and R. Miller, Spectra of structures and relations, to appear in the Journal of Symbolic Logic.

[14] D. R. Hirschfeldt, Prime models and relative decidability, Proceedings of the American Mathematical Society 134 (2006), pp. 1495-1498. MR2199197

[15] D. R. Hirschfeldt, B. Khoussainov, R. A. Shore and A. M. Slinko, Degree spectra and computable dimension in algebraic structures, Annals of Pure and Applied Logic 115 (2002), pp. 71-113. MR1897023 (2003d:03060)

[16] S. V. Ivanov, On the Burnside problem on periodic groups, Bulletin of the American Mathematical Society 27 (1992), pp. 257-260. MR1149874 (93a:20061)

[17] A. N. Khisamiev, On the upper semilattice $\mathcal{L}_{E}$, Siberian Mathematical Journal 45 (2004), pp. 173-187. MR2048764 (2005f:03061)

[18] J. F. Knight, Degrees coded in jumps of orderings, Journal of Symbolic Logic 51 (1986), pp. 1034-1042. MR0865929 (88j:03030)

[19] F. Levi and B. L. van der Waerden, Über eine besondere Klasse von Gruppen, Abhandlungen aus dem Mathematischen Seminar der Universität Hamburg 9 (1933), pp. 154-158. 
[20] R. C. Lyndon and P. E. Schupp, Combinatorial Group Theory, Springer-Verlag, Berlin, 1977. MR 0577064 (58:28182)

[21] P. S. Novikov and S. I. Adjan, Infinite periodic groups I, Izvestia Akademii Nauk SSSR, Ser. Math. 32 (1968), pp. 212-244 (in Russian). MR0240178 (39:1532a)

[22] P. S. Novikov and S. I. Adjan, Infinite periodic groups II, Izvestia Akademii Nauk SSSR, Ser. Math. 32 (1968), pp. 251-524 (in Russian). MR0240179 (39:1532b)

[23] P. S. Novikov and S. I. Adjan, Infinite periodic groups III, Izvestia Akademii Nauk SSSR, Ser. Math. 32 (1968), pp. 709-731 (in Russian). MR0240180(39:1532c)

[24] S. Oates, Jump Degrees of Groups, Ph.D. dissertation, University of Notre Dame, 1989.

[25] P. G. Odifreddi, Classical Recursion Theory, vol. II, Elsevier, Amsterdam, 1999. MR 1718169 (2001b:03040)

[26] M. O. Rabin, Computable algebra, general theory and theory of computable fields, Transactions of the American Mathematical Society 95 (1960), pp. 341-360. MR0113807 (22:4639)

[27] L. J. Richter, Degrees of structures, Journal of Symbolic Logic 46 (1981), pp. 723-731. MR0641486 (83d:03048)

[28] J. J. Rotman, The Theory of Groups, second edition, Allyn and Bacon, Boston, 1973. MR0442063(56:451)

[29] I. N. Sanov, Solution of Burnside's problem for exponent four, Leningrad State University Annals, Ser. 10 (1940), pp. 166-170 (in Russian). MR0003397(2:212c)

[30] T. A. Slaman, Relative to any nonrecursive set, Proceedings of the American Mathematical Society 126 (1998), pp. 2117-2122. MR1443408 (98h:03047)

[31] R. I. Soare, Recursively Enumerable Sets and Degrees, Springer-Verlag, Berlin, 1987. MR0882921 (88m:03003)

[32] S. Wehner, Enumerations, countable structures, and Turing degrees, Proceedings of the American Mathematical Society 126 (1998), pp. 2131-2139. MR1443415 (98h:03059)

Department of Mathematics, George Washington University, Washington, D.C. 20052

E-mail address: gdab@gwu.edu

Department of Mathematical Sciences, University of Texas at Dallas, Richardson, TEXAS 75083

E-mail address: mdab@utdallas.edu

Department of Mathematics, George Washington University, Washington, D.C. 20052

E-mail address: harizanv@gwu.edu

Department of Mathematics, State University of New York at Buffalo, Buffalo, New YorK 14260

E-mail address: asikora@buffalo.edu 